\title{
Kernos
}

Revue internationale et pluridisciplinaire de religion grecque antique

$16 \mid 2003$

Varia

\section{W.D. FURLEY, J.M. BREMMER, Greek Hymns}

\section{Danièle Aubriot-Sévin}

\section{(2) OpenEdition}

\section{Journals}

Édition électronique

URL : http://journals.openedition.org/kernos/847

DOI : $10.4000 /$ kernos. 847

ISSN : 2034-7871

Éditeur

Centre international d'étude de la religion grecque antique

Édition imprimée

Date de publication : 1 janvier 2003

Pagination : 374-375

ISSN : 0776-3824

Référence électronique

Danièle Aubriot-Sévin, «W.D. furley, J.M. Bremmer, Greek Hymns », Kernos [En ligne], 16 | 2003, mis en ligne le 14 avril 2011, consulté le 24 septembre 2020. URL : http://journals.openedition.org/kernos/ 847 ; DOI : https://doi.org/10.4000/kernos.847 
contenu de la syllogè, en une analogie intéressante avec les récits hagiographiques «à raconter ", legenda, ce qui le distingue adéquatement du «mythe ". Il est dès lors étonnant de voir apparaître le titre «Le mythe d'Arion » au chapitre VI, alors que le contexte d'énonciation du récit est identique. Mais le point est véniel... Ce parcours très bien mené et bien documenté se lit avec plaisir et permet de présager le meilleur pour la suite des travaux de cette jeune chercheuse.

Vinciane Pirenne-Delforge (FNRS - Université de Liège)

William D. Furley, Jan Maarten Bremer, Greek Hymns. I: The Texts in Translation. II: Greek Texts and Commentary, Tübingen, Mohr Siebeck, 2001. 2 vol. $15 \times 23 \mathrm{~cm}$, xxII +411 \& 443 p. (Studien und Texte zu Antike und Christentum, 10). ISBN : 3-16$147527-5$ \& 3-16-147553-4.

Par cette publication, l'intention des auteurs est de mettre à la disposition du public une sélection représentative des hymnes grecs (de l'âge classique - et même antérieur - à la période hellénistique), qui réunisse en une sorte de panorama aussi bien des textes issus des découvertes archéologiques ou papyrologiques des deux siècles écoulés, que des textes transmis par la tradition manuscrite «normale». Il s'agit donc d'une édition de quelque soixante-dix hymnes de provenances diverses.

L'ouvrage est conçu en deux volumes complémentaires, aptes à s'adresser éventuellement à des publics différents. En effet, le volume I se compose d'une introduction générale (pour dresser un bilan des connaissances concernant les hymnes grecs), suivie d'une traduction en vers et d'une notice plus ou moins développée sur chacun des hymnes choisis (son auteur, les circonstances de sa découverte, le genre auquel il appartient, ses qualités littéraires, etc.); ce tome est donc aisément accessible à tous ceux qui, sans être hellénistes, s'intéressent à l'histoire des religions, à l'anthropologie culturelle, à la théologie. Le volume II quant à lui s'adresse à une audience plus spécialisée : il offre le texte grec accompagné d'un apparat critique, et de tous les renseignements souhaitables concernant les éditions et études antérieures, l'analyse métrique, la datation probable; vient ensuite un commentaire serré vers par vers, aussi bien au plan de la langue qu'à celui du contenu. Cette présentation en deux volumes permet de lire commodément en regard le texte et sa traduction.

Les textes retenus pour cette sélection sont (dans chacun des deux volumes) répartis selon douze chapitres : les sept premiers classent les hymnes en fonction de la provenance géographique des sources épigraphiques et lyriques (Crète, Delphes, Délos, Lesbos et Ionie, Thèbes, Epidaure, Athènes); les quatre suivants examinent les sources théâtrales les chapitres huit, neuf et dix concernant respectivement chacun des trois tragiques (Eschyle : Suppl., Ag., Eum.; Sophocle : Ant., O.R., O.C.; Euripide : Hipp., Hclides, Ion, I.T.), le onzième Aristophane (Cav., Lysistr., Thesm., Gren.); enfin le douzième et dernier chapitre est consacré à cinq hymnes variés qui n'entraient pas dans les classifications précédentes. Si le volume I fait précéder les textes d'une introduction, le volume II les fait suivre d'appendices extrêmement précieux : sur les épithètes et attributs des dieux, sur les lieux sacrés (leurs noms et les termes servant à les décrire), sur l'accompagnement musical. Enfin chacun des deux tomes se clôt sur un index (un index général pour le premier; un index des noms et mots grecs judicieusement choisis pour le second), et chacun comporte une bibliographie. L'inconvénient de ces deux bibliographies est qu'elles se recoupent dans une assez large mesure. Mais l'avantage (qui prime amplement) est que chaque volume est autonome (à propos de la bibliographie du volume II, on peut observer que certains travaux mentionnés donnent l'impression de n'être pas toujours utilisés autant qu'ils auraient pu l'être : par ex. p. 29-30 de II, un mot sur le sens de $\lambda(\sigma \sigma o \mu a \iota$ n'aurait pas semblé superflu, et p. 116, en rester pour ápátav au LSS paraît un peu court; mais cette réserve ne concerne qu'un menu détail ponctuel). Pour l'un comme pour l'autre tome, on est sensible à l'étendue de l'information ainsi regroupée.

Les A, proposent donc dans cet ouvrage un large éventail d'anciens hymnes religieux qui nous restent de la Grèce, jusqu'à la période hellénistique. Ce qui fait le prix de cette publication est le rassemblement de témoignages jusqu'ici épars, ainsi que le parti de considérer sur le même plan textes épigraphiques et textes « littéraires ». Sans doute a-t-il 
fallu faire un choix, éliminer les pièces par trop lacunaires pour garder au livre un agrément de lecture qui le rende attrayant (ce qui est l'une de ses qualités, hautement appréciable). De même on comprend aisément que, pour demeurer dans des dimensions acceptables, il ait encore été nécessaire d'effectuer une sélection. Aussi les Hymmes bomériques et ceux de Callimaque, facilement accessibles par alleurs, ne sont-ils pas considérés dans cette publication - non plus que les hymnes orphiques. Tout cela était non seulement légitime, mais souhaitable. Ce qui en revanche n'est pas sans poser quelque problème, c'est que les témoignages non retenus ne reçoivent qu'une mince considération dans l'introduction, quoiqu'elle se veuille générale (organisée comme elle l'est en trois sections : nature des hymnes grecs, revue des textes subsistants, forme et composition des hymnes). Comme on peut s'y attendre, ce déséquilibre se répercute sur la bibliographie, où sont laissées de côté des études qui pourtant éclairent la littérature hymnique dans son ensemble. Ainsi les Hymnes orphiques sont-ils expédiés en six lignes (p. 49-50), sans que soit seulement mentionné l'article décisif (e.a. pour l'expérience cultuelle

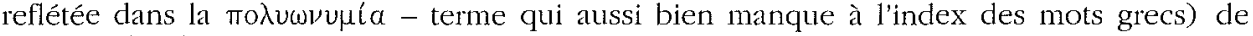
Jean Rudhardt : "Quelques réflexions sur les hymnes orphiques", in Ph. Borgeaud (éd.), Orpbisme et Orpbée. En l'bonneur de Jean Rudbardl, Genève, 1991 (Recbercbes et Rencontres, 3), p. 263-289. Les Hymnes bomériques reçoivent un peu plus d'attention (2 pages : 4143), mais il semble d'autant plus surprenant qu'on doive se passer d'analyses fondamentales comme celles de Claude Calame - si essentielles pour la réflexion sur les hymnes en général, et pour la reconsidération de la valeur proprement religieuse de certains textes «littéraires »: je mentionnerai surtout "L'hymne homérique à Déméter comme offrande: regard rétrospectif sur quelques catégories de l'anthropologie de la religion grecque", Kernos 10 (1997), p. 111-133; ou encore "Variations énonciatives, relations avec les dieux et fonctions poétiques dans les Hymnes homériques", $M H I 52$ (1995), p. 2-19, et "Rythme, voix et mémoire de l'écriture en Grèce classique", in R. Pretagostini (éd.), Tradizlone e innovazione nelle cultura greca da Omero all'età ellenislica. Scritti in Onore di Bruno Gentili, Rome, 1993, p. 785-799. De même, à la page 45 où il est question des hymnes de Callimaque, on aurait pu voir apparaître le nom d'André Hurst, "Contrepoints de Callimaque", MH 51 (1994), p. 150-163. Certes, redisons-le, les A. étaient assurément obligés de se limiter : ils indiquent (t. I, p. 40) que, parmi les textes subsistants, ils vont s'attacher surtout aux hymnes lyriques (sans s'interdire toutefois la présence, par exemple, d'un péan comme celui d'Isyllos à Asclépios, qui est composé en hexamètres : t. II, p. 183-4), à la condition qu'ils relèvent (effectivement ou fictivement) cle la religion cultuelle. Cela entraîne nécessairement que certaines orientations soient privilégiées au détriment d'autres, aperçues plus rapidement. Néanmoins l'absence des études mentionnées plus haut provoque une sorte de porte à faux qui peut gêner - précisément dans la perspective (qui est celle des A.) d'une reconsidération de la dichotomie traditionnelle entre hymnes littéraires et hymnes cultuels. En un mot non seulement ce ne sont pas tous les hymnes grecs qui sont concernés, mais du même coup la littérature hymnique est présentée sous un éclairage qui ne balaie pas tout le champ de la question pour la période concernée. Sans doute pareille ambition eût-elle été une gageure, impossible à tenir dans les conditions actuelles de l'édition; au reste, la formulation du titre se gardait d'afficher nettement cette prétention. Peut-être aurait-on aimé que cette réserve soit signifiée plus fermement, que ses conséquences soient indiquées avec plus de clarté. Mais l'essentiel est que ce qui est considéré ici le soit de manière admirable - et c'est le cas.

On peut donc estimer que les promesses sont tenues et que l'ouvrage, tel qu'il est, constitue une réussite certaine. La présentation est nette, la typographie claire, l'édition remarquablement soignée. Les commentaires se recommandent par leur clarté, leur sobriété, et la riche précision de leur information : on va droit au but, sans afféterie ni jargon. Le recueil de ces textes épars rendra assurément tous les services qu'en escomptent ses A. Nous avons là un travail dont, sans nul doute, la publication va marquer un pas décisif dans la diffusion des connaissances sur la religion grecque.

Danièle Aubriot (Université d'Amiens) 